\title{
A series of "cut" Bessel functions
}

\author{
By E. T. Copson and W. L. Ferrar.
}

(Received 8th January, 1938. Read 21st January, 1938.)

\section{$\S 1 . \quad$ Introduction.}

1.1. In Kottler's theoretical discussion ${ }^{1}$ of the diffraction of a plane wave of monochromatic light of wave-length $2 \pi / k$ by a black halfplane, the function

$$
f(r, \theta)=\frac{1}{2 \pi} \int_{0}^{\infty} e^{i k r \cosh t} \frac{\sin \theta}{\cosh t+\cos \theta} d t,
$$

where $(r, \theta, z)$ are cylindrical coordinates, plays an important part. In particular it is necessary to have asymptotic formulae for $f(r, \theta)$, valid when $r$ is either very large or very small compared with the wave-length.

Kottler obtains these asymptotic formulae by showing that

$$
\frac{1}{k} \frac{\partial f}{\partial r}+i \cos \theta f=-\frac{1}{4} \sin \theta H_{0}^{(1)}(k r)
$$

where, in the usual notation ${ }^{2}, H_{n}^{(1)}(\lambda)$ denotes the Bessel function $J_{n}(\lambda)+i Y_{n}(\lambda)$ of integral order $n$, and then making use of the known properties of $H_{0}^{(1)}(\lambda)$. Whilst this method gives correct results, it is difficult to place it on a sound basis.

1.2. The asymptotic formula valid as $r \rightarrow+\infty$ may be obtained by a direct method, well-known in the theory of Bessel functions. For if we make the substitution $\cosh t=1+i v$ and then rotate the path of integration through a right-angle, we obtain

$$
f(r, \theta)=\frac{1}{2 \pi} \exp \left\{i\left(k r+\frac{1}{4} \pi\right)\right\} \int_{0}^{\infty} e^{-k r v}\left(1+\frac{\sin \theta d v}{\cos \theta+i v) \sqrt{ }\left(2 v+i v^{2}\right)},\right.
$$

and an application of Watson's Lemma (W., 236) gives the required expansion. There does not appear to be any corresponding very simple way of obtaining the expansion valid when $r$ is small.

'Ann. der Phys. 71 (1923), 457.508 (in particular, pages 496 and 499). We have found it convenient to change the sign of $i$ throughout.

${ }^{2} \mathrm{G}$. N. Watson, A treatise on the theory of Bessel functions (Cambridge, 1922), 73. In later references, this work is cited as $\mathbf{W}$. 
1.3. The form of the differential equation $(1 \cdot 12)$ suggests another line of approach. If we substitute in this equation the formal series

$$
f(r, \theta)=\sum_{1}^{\infty} a_{n} H_{n: !}^{(1)}(k r),
$$

where $a_{n}$ is independent of $r$, and use the recurrence formula (W., $74(4)$ )

we find that

$$
2 \frac{d H_{n}^{(1)}(k r)}{d(k r)}=H_{n-1}^{(1)}(k r)-H_{n+1}^{(1)}(k r),
$$

$$
f(r, \theta)=\frac{1}{2 i} \sum_{1}^{\infty} e^{-i n n i} H_{n}^{(1)}(k r) \sin n \theta
$$

is a particular solution of $(1 \cdot 12)$.

The series (1.31) is not easy to handle near $r=0$ because $H_{n}^{(1)}(\mathrm{kr})$ is of the order of $r^{-n}$ as $r$ tends to zero; some other representation is needed. In the present note we use a series (viz. $(2 \cdot 12)$ infra) which is derived from (1·31) by omitting from each of the functions $H_{n}^{(1)}(k r)$ all negative powers of $r$. The sum of this series is discontinuous at $\theta= \pm \frac{1}{2} \pi$, and we are obliged to consider separately the two ranges $|\theta|<\frac{1}{2} \pi$ and $\frac{1}{2} \pi<|\theta|<\pi$.

The proofs of our results are less easy than one might expect, though the difficulty is perhaps due to the line of proof we have adopted.

Our methods can also be applied to obtain the expansion of a second integral considered by Kottler, viz.

$$
g(r, \theta)=\frac{1}{\pi} \int_{0}^{\infty} e^{i k r \cosh t} \frac{\cos \frac{1}{2} \theta \cosh \frac{1}{2} t}{\cosh t+\cos \theta} d t,
$$

as a series of "cut" Bessel functions, in this case of order $n+\frac{1}{2}$. The discussion of this is omitted as no essentially new ideas are involved.

§2. Enunciation of the main theorem: preliminary lemmas.

$2 \cdot 1$. Let $h_{n}^{(1)}(\lambda)$ denote the function obtained from the expansion of $H_{n}^{(1)}(\lambda)$ in the neighbourhood of $\lambda=0$ by omitting all the terms that involve negative powers of $\lambda$. It is usual to call a function of this sort a cut Bessel function.

We shall prove

Theorem 1. Let

$$
F(\lambda)=\frac{1}{2 \pi} \int_{0}^{\infty} e^{i \lambda \cosh t} \frac{\sin \theta}{\cosh t+\cos \theta} d t .
$$

Then if $\lambda>0$ and $-\frac{1}{2} \pi<\theta<\frac{1}{2} \pi$,

$$
F(\lambda)=\frac{1}{2 i} \sum_{n=1}^{\infty} e^{-\frac{3}{2} n \pi i} h_{n}^{(1)}(\lambda) \sin n \theta .
$$


The proof rests on certain subsidiary lemmas which are given in the following sub-sections. Apart from Lemma 1, these lemmas are of some analytical interest; the reader will need only the results of Lemmas 2 and 3 in order to follow the proof of Theorem 1.

We suppose throughout that $\lambda>0$ and, in $\$ 2$ and 3 , that $-\frac{1}{2} \pi<\theta<\frac{1}{2} \pi$.

2.2. Lemma 1. If $\Sigma a_{n}$ is a bounded series, then $\Sigma a_{n} x^{n}$ is uniformly bounded in $0 \leqq x \leqq 1 ;$ moreover, $\Sigma a_{n} x^{n}$ is convergent when $0<x<1$, and its sum $s(x)$ is bounded in this open interval.

Let $\quad \sigma_{n}=a_{0}+a_{1}+\ldots+a_{n}$.

Then $\quad a_{0}+a_{1} x+a_{2} x^{2}+\ldots+a_{n} x^{n}$

$$
=(1-x)\left(\sigma_{0}+\sigma_{1} x+\sigma_{2} x^{2}+\ldots+\sigma_{n-1} x^{n-1}\right)+\sigma_{n} x^{n} .
$$

But $\left|\sigma_{n}\right|<K$ for all $n$; hence, if $0<x<1$,

$$
\left|a_{0}+a_{1} x+\ldots+a_{n} x^{n}\right|<K\left(1-x^{n}\right)+K x^{n}=K .
$$

This proves the first part of the lemma.

By Dirichlet's test, the series $\sum a_{n} x^{n}$ is convergent when $0<x<1$; it follows at once that $|s(x)| \leqq K$ in this open interval.

Lemma 2. Let $\Sigma a_{n}$ be a bounded series, $s(x)$ the sum of $\Sigma a_{n} x^{n}$ when $0 \leqq x<1 ;$ let

Then

$$
\begin{gathered}
E(\lambda, x)=\exp \left\{\frac{1}{2} i \lambda\left(x+x^{-1}\right)\right\} \quad(x>0) . \\
\int_{0}^{1} s(x) E(\lambda, x) d x=\Sigma a_{n} \int_{0}^{1} x^{n} E(\lambda, x) d x .
\end{gathered}
$$

The proof of this lemma might almost be taken for granted. But. the fact that $s(1)$ and $E(\lambda, 0)$ are not defined raises minor difficulties, and we have thought it desirable to set out the proof.

Let $s_{n}(x)=a_{0}+a_{1} x+\ldots+a_{n} x^{n}$. Take an arbitrary positive. number $\epsilon$. Then, by Lemma 1 and the fact that $|E(\lambda, x)|=1$ when $0<x<1$, there exists a positive number $\delta$ such that, whenever $0<\delta^{\prime}<\delta$,

$$
\begin{aligned}
& \left|\int_{1-\delta}^{1-\delta^{\prime}}\left\{s(x)-s_{n}(x)\right\} E(\lambda, x) d x\right|<\frac{1}{\zeta} \epsilon, \\
& \left|\int_{\delta^{\prime}}^{\delta}\left\{\delta(x)-s_{n}(x)\right\} E(\lambda, x) d x\right|<\frac{1}{3 \zeta} \epsilon,
\end{aligned}
$$

independently of the value of $n$. Hence

$$
\int_{0}^{1}\left\{s(x)-s_{n}(x)\right\} E(\lambda, x) d x \equiv \int_{0}^{1} F_{n}(\lambda, x) d x,
$$


say, is defined as an improper integral. Moreover

$$
\left|\int_{0}^{\delta} F_{n}(\lambda, x) d x\right|+\mid \int_{1-\delta}^{1} F_{n}(\lambda, x) d x: \leqq 2 \epsilon,
$$

independently of the value of $n$.

But, by the uniform convergence of $\Sigma a_{n} x^{n}$ in $(\delta, 1-\delta)$ and the boundedness of $E(\lambda, x)$, there exists an integer $N$ such that, whenever $n \geqq N$,

$$
\left|\int_{\delta}^{1-\delta} F_{n}(\lambda, x) d x\right|<\frac{1}{3} \epsilon
$$

It now follows that

$$
\lim _{n \rightarrow \infty} \int_{0}^{1}\left\{s(x)-s_{n}(x)\right\} E(\lambda, x) d x=0 .
$$

Again, a modification of the argument used above shows that each of the integrals

$$
\int_{0}^{1} s(x) E(\lambda, x) d x, \quad \int_{0}^{1} x^{n} E(\lambda, x) d x
$$

is defined as an improper integral. Hence, by $(2 \cdot 23)$,

$$
\int_{0}^{1} s(x) E(\lambda, x) d x=\lim _{n \rightarrow \infty} \int_{0}^{1} s_{n}(x) E(\lambda, x) d x,
$$

and so the lemma is proved.

2.3. As a preliminary to our next lemma, we note that, by W., 78 (8), with $z=-i \lambda$, and $\mathbf{W}_{\text {., }} 80(15)$, the terms in the expansion of $H_{n}^{(1)}(\lambda)$ near $\lambda=0$ which involve negative powers of $\lambda$ are given by

$$
-\frac{i}{\pi} \sum_{m=0}^{[1 .(n-1)]} \frac{(n-m-1) !}{m !\left(\frac{1}{2} \lambda\right)^{n-2 m}}=-\frac{i}{\pi} S_{n}(\lambda),
$$

where $S_{n}(\lambda)$ is Schläfli's polynomial, as defined, for example, in W., $285(1)$. Hence the "cut" Bessel function $h_{n}^{(1)}(\lambda)$ is given by

$$
h_{n}^{(1)}(\lambda)=H_{n}^{(1)}(\lambda)+\frac{i}{\pi} S_{n}(\lambda) .
$$

Now, by W., $288(2)$ with $a=0$, we have

$$
\begin{aligned}
S_{n}(\lambda) & =\int_{0}^{\infty}\left\{e^{n \theta}-e^{-n(\pi i+\theta)}\right\} e^{-\lambda \sinh \theta} d \theta \\
& =2 e^{-\frac{1}{2} n \pi i} \int_{\frac{\underline{\underline{b}} \pi i}{2} \pi i+\infty}^{\ln } \sinh n \phi e^{i \lambda \cosh \phi} d \phi,
\end{aligned}
$$

on writing $\theta=\phi-\frac{1}{2} \pi i$. Moreover, by W., 180, (8),

$$
H_{n}^{(1)}(\lambda)=\frac{2}{\pi i} e^{-\frac{1}{2} n \pi i} \int_{0}^{\frac{1}{3} \pi i+\infty} \cosh n \phi e^{i \lambda \cosh \phi} d \phi .
$$


From $(2 \cdot 31)$ and $(2 \cdot 32)$ follows

Lemma 3. The cut Bessel function $h_{n}^{(1)}(\lambda)$ is given by

$$
\frac{1}{2} \pi i e^{\frac{\jmath}{n \pi i}} h_{n}^{(1)}(\lambda)=\int_{0}^{\frac{1}{3} \pi i} \cosh n \phi e^{i \lambda \cosh \phi} d \phi+\int_{\frac{1}{2 \pi i}}^{\not \pi r i+\infty} e^{-n \phi} e^{i \lambda \cosh \phi} d \phi .
$$

\section{$\S 3 . \quad$ Proof of Theorem 1.}

$3 \cdot 1$. If in the definition $(2 \cdot 11)$ we put $e^{-t}=x$ and use the notation $E(\lambda, x)$ of Lemma 2, we obtain

$$
F(\lambda)=\frac{1}{\pi} \int_{0}^{1} E(\lambda, x) \frac{\sin \theta}{x^{2}+2 x \cos \theta+1} d x .
$$

But when $0 \leqq x<1$,

$$
\frac{\sin \theta}{x^{2}+2 x \cos \theta+1}=-\sum_{n=1}^{\infty} x^{n-1} \sin (n \theta+n \pi)
$$

moreover, for any fixed value of $\theta$,

$$
\Sigma \sin (n \theta+n \pi)
$$

is a bounded series. Hence, by Lemma 2,

$$
F(\lambda)=-\frac{1}{\pi} \sum_{n=1}^{\infty}(-1)^{n} \sin n \theta \int_{0}^{1} E(\lambda, x) x^{n-1} d x,
$$

or, on restoring the original variable of integration,

$$
F(\lambda)=-\frac{1}{\pi} \sum_{n=1}^{\infty}(-1)^{n} \sin n \theta \int_{0}^{\infty} e^{i \lambda \cosh t} e^{-n t} d t .
$$

3.2. Now if $n \geqq 0$, we have, by Cauchy's theorem,

$$
\int_{0}^{\infty} e^{i \lambda \cosh t} e^{-n t} d t=\int_{0}^{3 \pi i} e^{i \lambda \cosh t} e^{-n t} d t+\int_{\frac{d}{3 \pi i}}^{3 \pi i+\infty} e^{i \lambda \cosh t} e^{-n t} d t .
$$

The integral over $\left(\frac{1}{2} \pi i, \frac{1}{2} \pi i+\infty\right)$ in $(3 \cdot 21)$ tallies with that in Lemma 3 , whereas that over $\left(0, \frac{1}{2} \pi i\right)$ does not. We discuss the integral over $\left(0, \frac{1}{2} \pi i\right)$ in the next sub-section.

$3 \cdot 3$. Let us consider the integral ${ }^{1}$.

$$
\begin{aligned}
I & =\int_{0}^{i \pi} e^{i \lambda \cos y} \frac{i \sin \theta}{\cos y+\cos \theta} d y \\
& =\int_{0}^{i \pi} e^{i \lambda \cos y}\left\{\frac{e^{i \theta}}{e^{i y}+e^{i \theta}}-\frac{e^{-i \theta}}{e^{i y}+e^{-i \theta}}\right\} d y \\
& =\lim _{r \rightarrow 1-0} \int_{0}^{l i \pi} e^{i \lambda \cos y}\left\{\frac{e^{i \theta}}{r e^{i y}+e^{i \theta}}-\frac{e^{-i \theta}}{r e^{i y}+e^{-i \theta}}\right\} d y .
\end{aligned}
$$

1 The device used here was suggested by a more lengthy proof of the theorem on different lines. 
The last step is justified by the fact that the integrand in $(3 \cdot 32)$ is a continuous function of both variables $r$ and $y$ on account of the restriction $-\frac{1}{2} \pi<\theta<\frac{1}{2} \pi$. Expanding in powers of $r$, we have

$$
\begin{aligned}
I & =\lim _{r \rightarrow 1-0} \int_{0}^{\frac{1}{3} \pi} e^{i \lambda \cos y} \sum_{n=1}^{\infty}(-2 i \sin n \theta)(-r)^{n} e^{n i y} d y \\
& =\lim _{r \rightarrow 1-0}(-2 i) \sum_{n=1}^{\infty}(-r)^{n} \sin n \theta \int_{0}^{\frac{i}{2 \pi}} e^{i \lambda \cos y} e^{n i y} d y .
\end{aligned}
$$

The series obtained by putting $r=1$ is convergent, as an integration by parts beginning with

$$
\frac{1}{n i} \int_{0}^{\frac{1}{2} \pi} e^{i \lambda \cos y} d\left(e^{n i y}\right)
$$

will show. Hence, by Abel's theorem on the continuity of power series,

$$
\begin{aligned}
I & =-2 i \sum_{n=1}^{\infty}(-1)^{n} \sin n \theta \int_{0}^{\frac{\lambda}{2} \pi} e^{i \lambda \cos y} e^{n i y} d y \\
& =-2 \sum_{n=1}^{\infty}(-1)^{n} \sin n \theta \int_{0}^{\frac{1}{2 \pi i}} e^{i \lambda \cosh t} e^{n t} d t
\end{aligned}
$$

But we also have

$$
\frac{i \sin \theta}{\cos y+\cos \theta}=\lim _{r \rightarrow 1}\left\{\frac{e^{i \theta}}{r e^{-i y}+e^{i \theta}}-\frac{e^{-i \theta}}{r e^{-i y}+e^{-i \theta}}\right\},
$$

and so we can carry out the previous transformations with $e^{-i y}$ in place of $e^{i y}$. It then follows that

$$
I=-2 \sum_{n=1}^{\infty}(-1)^{n} \sin n \theta \int_{0}^{\frac{1}{2} \pi i} e^{i \lambda \cosh t} e^{-n t} d t .
$$

Combining $(3 \cdot 33)$ and $(3 \cdot 34)$, we have

$$
\begin{aligned}
\sum_{n=1}^{\infty}(-1)^{n} & \sin n \theta \int_{0}^{\frac{1}{2} \pi i} e^{i \lambda \cosh t} e^{-n t} d t \\
= & \sum_{n=1}^{\infty}(-1)^{n} \sin n \theta \int_{0}^{\frac{1}{2} \pi i} e^{i \lambda \cosh t} \cosh n t d t
\end{aligned}
$$

$3 \cdot 4$. By $(3 \cdot 11),(3 \cdot 21)$ and $(3 \cdot 35)$, we now have

$$
F(\lambda)=-\frac{1}{\pi} \sum_{n=1}^{\infty}(-1)^{n} \sin n \theta G(n, \lambda),
$$

where $G(n, \lambda)=\int_{0}^{\frac{1}{2} \pi i} e^{i \lambda \cosh t} \cosh n t d t+\int_{\frac{1}{2 \pi i}}^{\frac{1}{2} \pi i+\infty} e^{i \lambda \cosh t} e^{-n t} d t$.

But, by Lemma 3,

$$
G(n, \lambda)=\frac{1}{2} \pi i e^{\frac{1}{2} n \pi i} h_{n}^{(1)}(\lambda)
$$


Hence

$$
F(\lambda)=\frac{1}{2 i} \sum_{n=1}^{\infty} e^{-\frac{t}{2} n \pi i} h_{n}^{(1)}(\lambda) \sin n \theta .
$$

But this is the required equation $(2 \cdot 12)$, and so Theorem 1 is proved.

\section{§4. Extensions of Theorem 1 .}

4*1. The argument of $\S 3 \cdot 3$ fails when $\theta= \pm(\pi-\alpha)$ and $0<\alpha<\frac{1}{2} \pi$, since the integral $(3 \cdot 31)$ exists only as a Cauchy principal value on account of the singularity at $y=a$; moreover this principal value is not equal to $(3 \cdot 32)$ or to the corresponding limit with $r e^{-i y}$ replacing $r e^{i y}$. It turns out that equation (3.35) no longer holds and has to be replaced by the identity of

Lemma 4. If $\frac{1}{2} \pi<|\theta|<\pi$, then

$$
\begin{aligned}
& -\sum_{n=1}^{\infty}(-1)^{n} \sin n \theta \int_{0}^{\frac{1}{2} \pi i} e^{i \lambda \cosh t} e^{-n t} d t \\
& \quad= \pm \frac{1}{2} \pi e^{-i \lambda \cos \theta}-\sum_{n=1}^{\infty}(-1)^{n} \sin n \theta \int_{0}^{\frac{1}{3} \pi i} e^{i \lambda \operatorname{cosb} t} \cosh n t d t
\end{aligned}
$$

where the upper or lower sign is taken according as $\theta$ is positive or negative.

Let us suppose first that $\theta=\pi-\alpha$ where $0<\alpha<\frac{1}{2} \pi$, and consider

$$
\int e^{i \lambda \cos z} \frac{i \sin \alpha}{\cos z-\cos \alpha} d z
$$

along two paths both beginning at $z=0$ and ending at $z=\frac{1}{2} \pi$. The first of these paths, $\Gamma_{1}$ say, lies in the lower half-plane, the other, $\Gamma_{2}$ say, in the upper half-plane. Then, by Cauchy's theorem of residues,

$$
\int_{\Gamma_{1}}-\int_{\Gamma_{2}}=2 \pi i(\text { residue at } z=a)=2 \pi e^{i \lambda \cos a}
$$

Now the integral along $\Gamma_{2}$ is equal to

$$
\begin{gathered}
\lim _{r \rightarrow 1-0} \int_{\Gamma_{2}} e^{i \lambda \cos z}\left\{\frac{e^{i a}}{r e^{i z}-e^{i a}}-\frac{e^{-i a}}{r e^{i z}-e^{-i \alpha}}\right\} d z \\
=\lim _{r \rightarrow 1-0} \int_{\Gamma_{z}} 2 i e^{i \lambda \cos z} \sum_{n=1}^{\infty} r^{n} e^{n i z} \sin n a d z \\
=\lim _{r \rightarrow 1-0} \sum_{n=1}^{\infty} 2 i r^{n} \sin n a \int_{\Gamma_{z}} e^{i \lambda \cos z} e^{n i z} d z,
\end{gathered}
$$

term-by-term integration being valid since $\left|r e^{i z}\right| \leqq r$ on $\Gamma_{2}$. An 
application of Cauchy's theorem enables us to replace $\Gamma_{2}$ by a segment of the real axis, and so

$$
\begin{aligned}
\int_{\Gamma_{2}} & =\lim _{r \rightarrow 1-0} \sum_{n=1}^{\infty} 2 i r^{n} \sin n \alpha \int_{0}^{\frac{1}{2} \pi} e^{i \lambda \cos x} e^{n i x} d x \\
& =\lim _{r \rightarrow 1-0} \sum_{n=1}^{\infty} 2 r^{n} \sin n \alpha \int_{0}^{\frac{1}{2 \pi i}} e^{i \lambda \cosh t} e^{n t} d t .
\end{aligned}
$$

Hence, by Abel's theorem on the continuity of power series, ${ }^{1}$

$$
\int_{\Gamma_{z}}=\sum_{n=1}^{\infty} 2 \sin n a \int_{0}^{\frac{1}{2} \pi i} e^{i \Lambda \cosh t} e^{n t} d t .
$$

By a similar argument, it follows from the identity

that

$$
\int_{\Gamma_{1}}=\lim _{r \rightarrow 1-0} \int_{\Gamma_{1}} e^{i \lambda \cos z}\left\{\frac{e^{i \alpha}}{r e^{-i z}-e^{i \alpha}}-\frac{e^{-i \alpha}}{r e^{-i z}-e^{-i \alpha}}\right\} d z
$$

$$
\int_{\Gamma_{1}}=\sum_{n=1}^{\infty} 2 \sin n \alpha \int_{0}^{j \pi i} e^{i \lambda \cosh t} e^{-n t} d t .
$$

From equations $(4 \cdot 12),(4 \cdot 13),(4 \cdot 14)$, we easily obtain $(4 \cdot 11)$ for the case $\frac{1}{2} \pi<\theta<\pi$. The result when $-\pi<\theta<-\frac{1}{2} \pi$ follows by changing the sign of $\theta$.

4.2. If we repeat the argument of $\S 3$, using $(4 \cdot 11)$ instead of $(3 \cdot 35)$, we obtain

THEOREM 2. Let

$$
F(\lambda)=\frac{1}{2 \pi} \int_{0}^{\infty} e^{i \lambda \cosh t} \frac{\sin \theta}{\cosh t+\cos \theta} d t .
$$

Then if $\lambda>0$ and $\frac{1}{2} \pi<|\theta|<\pi$,

$$
F(\lambda)= \pm \frac{1}{2} e^{-i \lambda \cos \theta}+\frac{1}{2 i} \sum_{n=1}^{\infty} e^{-\frac{i}{2} n i i} h_{n}^{(1)}(\lambda) \sin n \theta,
$$

where the upper or lower sign is taken according as $\theta$ is positive or negative.

4:3. By Lemma 3, we have

$$
h_{n}^{(1)}(\lambda)=\frac{2}{n \pi i} \cos \frac{n \pi}{2} e^{-\frac{1}{2} n \pi i}+O\left(\frac{1}{n^{2}}\right)
$$

for any fixed value of $\lambda$. Hence the series

$$
\sum_{n=1}^{\infty} e^{-\frac{1}{2} n \pi i} h_{n}^{(1)}(\lambda) \sin n \theta
$$

] $O f$. the argument which gave equation (3.33). 
converges for all values of $\theta$. But the integral $(2 \cdot 11)$ is a continuous function of $\theta$ when $|\theta|<\pi$; a comparison of $(2 \cdot 12)$ and (4.21) then shows that the sum of the series $(4 \cdot 32)$ is discontinuous at $\theta= \pm \frac{1}{2} \pi$.

Now by $(4 \cdot 31)$ and the Riesz-Fischer Theorem, ${ }^{1}(4 \cdot 32)$ is the Fourier series of an odd function $\Phi(\theta)$ of the class $L^{2}$, and evidently

$$
\begin{array}{rlrl}
\Phi(\theta) & =F & & \left(0<\theta<\frac{1}{2} \pi\right), \\
& =F-\frac{1}{2} e^{-i \lambda \cos \theta} & \left(\frac{1}{2} \pi<\theta<\pi\right) .
\end{array}
$$

But since $(4 \cdot 32)$ is convergent,

$$
\begin{aligned}
\sum_{n=1}^{\infty} e^{-\frac{1}{2} n \pi i} h_{n}^{(1)}(\lambda) \sin n \theta & =\lim _{r \rightarrow 1-0} \sum_{n=1}^{\infty} r^{n} e^{-\frac{1}{2} n \pi i} h_{n}^{(1)}(\lambda) \sin n \theta \\
& =\lim _{r \rightarrow 1-0} \frac{1}{2 \pi} \int_{0}^{2 \pi} \frac{1-r^{2}}{1-2 r \cos (\theta-\phi)+r^{2}} \Phi(\phi) d \phi \\
& =\frac{1}{2}\{\Phi(\theta+0)+\Phi(\theta-0)\}
\end{aligned}
$$

whenever the latter limit exists. ${ }^{2}$

In particular, putting $\theta= \pm \frac{1}{2} \pi$, we obtain

Theorem 3. If $\lambda>0$ and $\theta= \pm \frac{1}{2} \pi$,

$$
F(\lambda)= \pm \frac{1}{4} e^{-i \lambda \cos \theta}+\frac{1}{2 i} \sum_{n=1}^{\infty} e^{-\underline{i n} n i} h_{n}^{(1)}(\lambda) \sin n \theta
$$

where the upper or lower sign is taken according as $\theta$ is positive or negative.

$\S 5$. The behaviour of $F(\lambda)$ when $\lambda \rightarrow 0$ or $\infty$.

5·1. From Lemma 3, we have

$$
\lim _{\lambda \rightarrow 0} h_{n}^{(1)}(\lambda)=\frac{2}{n \pi i} \cos \frac{n \pi}{2} e^{-\frac{1}{2} n \pi i}
$$

Hence it follows from Theorem 1 that, if $|\theta|<\frac{1}{2} \pi$,

and so

$$
\lim _{\lambda \rightarrow 0} F(\lambda)=\frac{1}{2 \pi}\left\{\sin 2 \theta-\frac{1}{2} \sin 4 \theta+\frac{1}{3} \sin 6 \theta-\ldots\right\},
$$

$$
\lim _{\lambda \rightarrow 0} F(\lambda)=\frac{\theta}{2 \pi}
$$

${ }^{1}$ See, for example, Titchmarsh, Theory of Functions (Oxford, 1932), 423-4.

2 We have used here two well-known results in the theory of Fourier series. See, for example, Titchmarsh, loc. cit., 440, Exx. 6, 7. 
That the equation $(5 \cdot 11)$ holds in the wider range $|\theta|<\pi$ may be proved by means of Theorems 2 and 3, or, more directly, from the integral definition of $F(\lambda)$ by means of the calculus of residues.

Having obtained $(5 \cdot 11)$, we can show from the expansion of $h_{n}^{(1)}(\lambda)$ near $\lambda=0$ that, if $|\theta|<\pi$,

$$
F(\lambda)=\frac{\theta}{2 \pi}-\frac{i \sin \theta}{2 \pi} \lambda \log \lambda+O(\lambda)
$$

as $\lambda \rightarrow 0$.

5.2. For completeness we observe that, if $|\theta|<\pi$,

$$
2 \pi F(\lambda) \sim \sqrt{ }\left(\frac{1}{2} \pi / \lambda\right) \tan \frac{1}{2} \theta e^{i(\lambda+\pi / 4)}
$$

as $\lambda \rightarrow \infty$, a result best proved by the method of $\S 1 \cdot 2$.

University College, Dundee.

Hertford College, Oxford. 